\title{
Genomic identification of group A bZIP transcription factors and their responses to abiotic stress in carrot
}

\author{
F. Que, G.L. Wang, Y. Huang, Z.S. Xu, F. Wang and A.S. Xiong \\ State Key Laboratory of Crop Genetics and Germplasm Enhancement, \\ College of Horticulture, Nanjing Agricultural University, Nanjing, China \\ Corresponding author: A.S. Xiong \\ E-mail: xiongaisheng@njau.edu.cn
}

Genet. Mol. Res. 14 (4): 13274-13288 (2015)

Received May 12, 2015

Accepted August 13, 2015

Published October 26, 2015

DOI http://dx.doi.org/10.4238/2015.October.26.24

ABSTRACT. The basic-region/leucine-zipper (bZIP) family is one of the major transcription factor (TF) families associated with responses to abiotic stresses. Many members of group A in this family have been extensively examined and are reported to perform significant functions in ABA signaling as well as in responses to abiotic stresses. In this study, 10 bZIP factors in carrot were classified into group A based on their DNA-binding domains. The cis-acting regulatory elements and folding states of these 10 factors were analyzed. Evolutionary analysis of the group A members suggested their importance during the course of evolution in plants. In addition, cis-acting elements and the folding state of proteins were important for DNA binding and could affect gene expression. Quantitative RT-PCR was conducted to investigate the stress response of 10 genes encoding the group $A$ factors. Six genes showed responses to abiotic stresses, while four genes showed other special phenomenon. The current analysis on group A bZIP family TFs in carrot is the first to investigate the TFs of Apiaceae via genome analysis. These results provide new information for future studies on carrot.

Key words: bZIP; Group A; Transcription factor; Evolution; Abiotic stress; Carrot 


\section{INTRODUCTION}

Abiotic stress has emerged as an important factor affecting the growth and development of vegetable crops and limiting their yield (Vinocur and Altman, 2005). Among the various environmental factors, soil salinization, low and high temperatures, and drought are the main stressors for plant growth. For their survival, plants have evolved several mechanisms to resist these stresses (Vinocur and Altman, 2005). Previous studies have revealed that transcription factors (TFs) participate in plant's responses to abiotic stress (Chen et al., 2002).

Of the numerous TFs present in plants, seven major TF families have been reported to participate in such responses (Finkelstein and Lynch, 2000). The basic-region/leucine-zipper (bZIP) TF family, for example, performs significant functions in resisting abiotic stresses (Yáñez et al., 2009). The bZIP TFs are one of the most conserved proteins present in all eukaryotes (Riechmann et al., 2000). The bZIP domain of bZIP TFs is highly conserved and harbors a DNA-binding basic region as well as a leucine (Leu) zipper dimerization motif (Wang et al., 2011). The DNA-binding basic region is highly conserved and is used for binding DNA in a sequence-specific manner. The Leu zipper dimerization motif, which is not conserved, has the property to dimerize (Ellenberger et al., 1992). Based on their DNA-binding domains, 75 bZIP TFs in Arabidopsis have been classified into 10 groups of which 13 have been assigned to group A (Jakoby et al., 2002). A number of bZIP family TFs have been reported to be involved in abscisic acid (ABA)-mediated stress signaling (Fujita et al., 2005). ABA performs an important function in the response of plants to abiotic stress (Fujita et al., 2011).

In Arabidopsis, many members of the group A bZIP family TFs have been designated as ABA-responsive element (ABRE) -binding-factors (ABFs) or ABRE-binding-proteins (AREBs) (Finkelstein and Lynch, 2000; Jakoby et al., 2002). A number of studies have evaluated the function of group A TFs in rice, their results demonstrating that the overexpression of OsABI5 lead to a higher sensitivity to salt stress (Zou et al., 2008), and that of OsABF1 and OsABF2 enhanced the abiotic stress signaling (Hossain et al., 2010a,b). In Arabidopsis, the roles of AREB1 and AREB2 in response to ABA, drought, and high salt stresses have been well-examined (Hsieh et al., 2010). Although several reports on bZIP family TFs in Arabidopsis and rice are available, similar studies in carrot are lacking. Transcriptional regulation of group A TFs under abiotic stresses has not been sufficiently investigated in carrot.

Carrot (Daucus carota L.) is a biennial plant with the highest cultivation area among Apiaceae species. Molecular studies on this significant vegetable, best known for its rich nutrient content, are limited. In the present study, 75 bZIP family TFs were identified in carrot; ten members were classified under group A, based on carrot genome data (Xu et al., 2014). The bZIP motifs of the 10 genes, their upstream cis-regulatory elements, and their folding states were also analyzed. The expression profiles of the 10 genes, under 4 different environment stimuli (i.e., drought, heat, cold, and, salt) were analyzed to evaluate the responses of DcbZIPA genes to abiotic stresses. The results could help in elucidating the function of DcbZIPA genes in stress response as well as the structure of bZIP genes in carrot.

\section{MATERIAL AND METHODS}

\section{Plant materials, growth conditions, and treatments}

The carrot cultivar 'Kurodagosun' was used as the experimental material. This cultivar originated in Japan. It has cylindrical roots and shows high tolerance to heat stress. The plantlets 
were grown in an artificial climate chamber at Nanjing Agricultural University $\left(32^{\circ} 02^{\prime} \mathrm{N}, 118^{\circ} 50^{\prime} \mathrm{E}\right)$. Two-month-old plantlets were subjected to high $\left(38^{\circ} \mathrm{C}\right)$ and low $\left(4^{\circ} \mathrm{C}\right)$ temperature, high salt $(0.2 \mathrm{M}$ $\mathrm{NaCl})$, and dehydration (200 g/L PEG) conditions. The plant samples were collected at 0, 1, 2, 4, 8 , and $12 \mathrm{~h}$, after the treatment. The whole treated plants were harvested, frozen in liquid nitrogen, and stored at $-70^{\circ} \mathrm{C}$ for further study.

\section{Sequence database searches}

Sequences of the Arabidopsis bZIP TF family were obtained from The Arabidopsis Information Resource (http://www.arabidopsis.org/) while those of the carrot were downloaded from CarrotDB, which is a genomic and transcriptomic database of $D$. carota (http://apiaceae.njau. edu.cn/carrotdb/index.php; Xu et al., 2014).

\section{Motif recognition and phylogenetic analysis}

Motifs of the selected genes were analyzed using the MEME (Version 4.9.1) suite (Bailey et al., 2006). Multiple alignments of bZIP protein sequences were performed by Clustal X 1.83 software (Thompson et al., 1997) and the phylogenetic tree was constructed with MEGA 5.0 (Tamura et al., 2011).

\section{cis-Regulatory element analysis and prediction of folding state}

Putative cis-acting regulatory elements were discovered using the plant database PlantCARE (Lescot et al., 2002). The lengths of the upstream sequence for study ranged from 900 to $1500 \mathrm{bp}$. Predictions were performed employing the Foldlndex program (http://bioportal. weizmann.ac.il/fldbin/findex) (Prilusky et al., 2005).

\section{RNA isolation and relative quantitative real-time (qRT)-PCR analysis}

Total RNA was extracted using an RNA kit (Tiangen, Beijing, China) according to the manufacturer instructions. RNA from each of the treated samples was reverse transcribed into cDNA using the Prime ScriptRT reagent kit (TaKaRa, Dalian, China).

The primers for the selected DCbZIPA genes were designed using Primer Premier 5.0 software. Tubulin gene of carrot was selected as the internal control. Sequences of all the primers used in this study are provided in Table1. The primers were synthesized by Genscript Nanjing Inc. (Nanjing, China). qRT-PCR was performed in an ABI7300 qRT-PCR system (Applied Biosystems, Foster City, CA, USA) using a SYBR Premix Ex Taq kit (TaKaRa, Dalian, China) with the following reaction conditions: $95^{\circ} \mathrm{C}$ for $30 \mathrm{~s}$ followed by 40 cycles of $95^{\circ} \mathrm{C}$ for $5 \mathrm{~s}$ and $60^{\circ} \mathrm{C}$ for $30 \mathrm{~s}$ for annealing and extension. Melting curve analysis was performed at $65^{\circ} \mathrm{C}$ for $10 \mathrm{~s}$ ( 61 cycles) to test the specificity of amplification. The experiments were repeated thrice using independent RNA samples.

\section{RESULTS}

\section{Phylogenetic analysis of bZIP TF family in carrot}

To analyze the bZIP TF family in carrot, a phylogenetic tree was constructed using the 
amino acid sequences of 68 proteins from Arabidopsis and 75 proteins from carrot. In the tree, the 75 DcbZIP TFs were arranged in 10 subfamilies (A-I, and S), according to the classification of the bZIP family in Arabidopsis (Figure 1). We analyzed the constituents of each subfamily (group) of the bZIP in carrot, based on this phylogenetic tree. This included Group A, the third-largest group in the family (13\%) with 10 members and Group S, which included $31 \%$ of all bZIP family members and was the largest (Figure 2).

Table 1. Primer sequences for qRT-PCR amplification of Tubulin and 10 genes from group A bZIP TFs in carrot.

\begin{tabular}{|c|c|c|}
\hline Gene number & Gene name & Oligonucleotide sequences \\
\hline \multirow[t]{2}{*}{ DcbZIPA1 } & Dck13424-Forward primer & 5'-AATTCTAGTTTTGGAATTGGAT-3' \\
\hline & Dck13424-Reverse primer & 5'-GGTCTCTGTTGTAGTTGTGGCT-3' \\
\hline \multirow[t]{2}{*}{ DcbZIPA2 } & Dck24387-Forward primer & 5'-CAATGGTGGATATTATGGTGAG-3' \\
\hline & Dck24387-Reverse primer & 5'-TACAGTTGCTTGTTTGGGGAAA-3' \\
\hline \multirow[t]{2}{*}{ DcbZIPA3 } & Dck21551-Forward primer & 5'-ATTCTTGGTTAAGGCGGGTGTG-3' \\
\hline & Dck21551-Reverse primer & 5'-GCATTATCTGTTGCTGAGTTGG-3' \\
\hline \multirow[t]{2}{*}{ DcbZIPA4 } & Dck19713-Forward primer & 5'-TTTGGTGAGATGACTGTGGAGG-3' \\
\hline & Dck19713-Reverse primer & 5'-TGTGGGGAACTTTGTTGTGGGA-3' \\
\hline \multirow[t]{2}{*}{ DcbZIPA5 } & Dck70030-Forward primer & 5'-TTGACGAGGTGTGGCAGGATAT-3' \\
\hline & Dck70030-Reverse primer & 5'-TGCGGAGCACTTTGTTGAGGTA-3' \\
\hline \multirow[t]{2}{*}{ DcbZIPA6 } & Dck73230-Forward primer & 5'-CCGTGCCTACTGTGCCACACAT-3' \\
\hline & Dck73230-Reverse primer & 5'-GCTGGAACACTCAGCGATCCTT-3' \\
\hline \multirow[t]{2}{*}{ DcbZIPA7 } & Dck03542-Forward primer & 5'-CATGAATCCACAATATCACTAT-3' \\
\hline & Dck03542-Reverse primer & 5'-TACCCCTGCCTTGACCAAAAAA-3' \\
\hline \multirow[t]{2}{*}{ DcbZIPA8 } & Dck02510-Forward primer & 5'-ACAGATTTTCAGGGGAGGGTTA-3' \\
\hline & Dck02510-Reverse primer & 5'-TCAAGAGTGAGGGAATGGATTG-3' \\
\hline \multirow[t]{2}{*}{ DcbZIPA9 } & Dck48330-Forward primer & 5'-CCTTTGGGGAAATATAATAAGC-3' \\
\hline & Dck48330-Reverse primer & 5'-GGGCGGCAAGAGGAGAGTTGGG-3' \\
\hline \multirow[t]{2}{*}{ DcbZIPA10 } & Dck27084-Forward primer & 5'-GAATCTCCAAGAATCTCTAGGC-3' \\
\hline & Dck27084-Reverse primer & 5'-TAATCATTTTCACGGGAACACC-3' \\
\hline \multirow[t]{2}{*}{ Tubulin } & Tubulin-Forward primer & 5'-GAGTGGAGTTACCTGCTGCCTTC-3' \\
\hline & Tubulin-Reverse primer & 5'-ATGTAGACGAGGGAACGGAATCAAG-3' \\
\hline
\end{tabular}

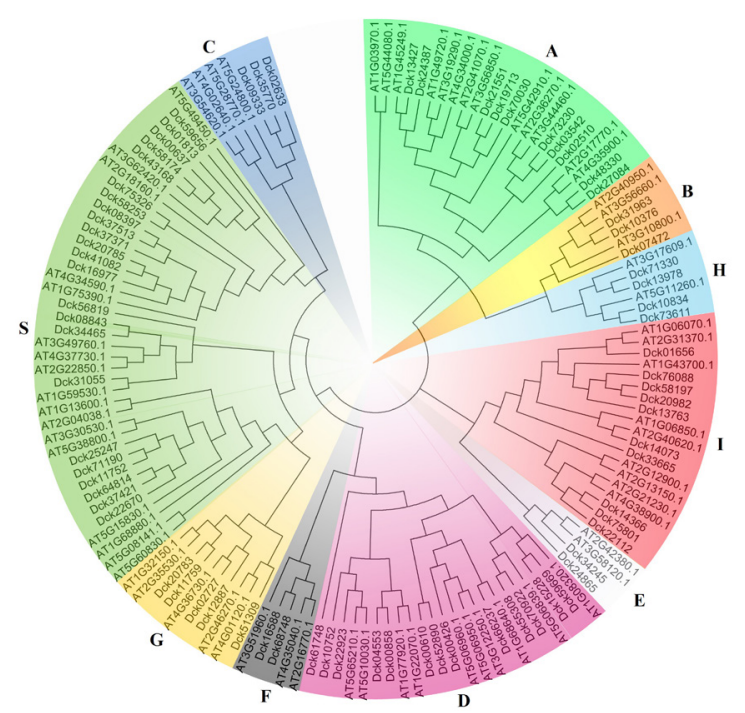

Figure 1. Phylogenetic tree of carrot and Arabidopsis bZIP family TFs. A total of 75 carrot and 68 Arabidopsis bZIP domain protein sequences were aligned by Clustal X 1.83, and the phylogenetic tree was constructed via MEGA5.0. Backgrounds with different colors represent different subfamilies. 


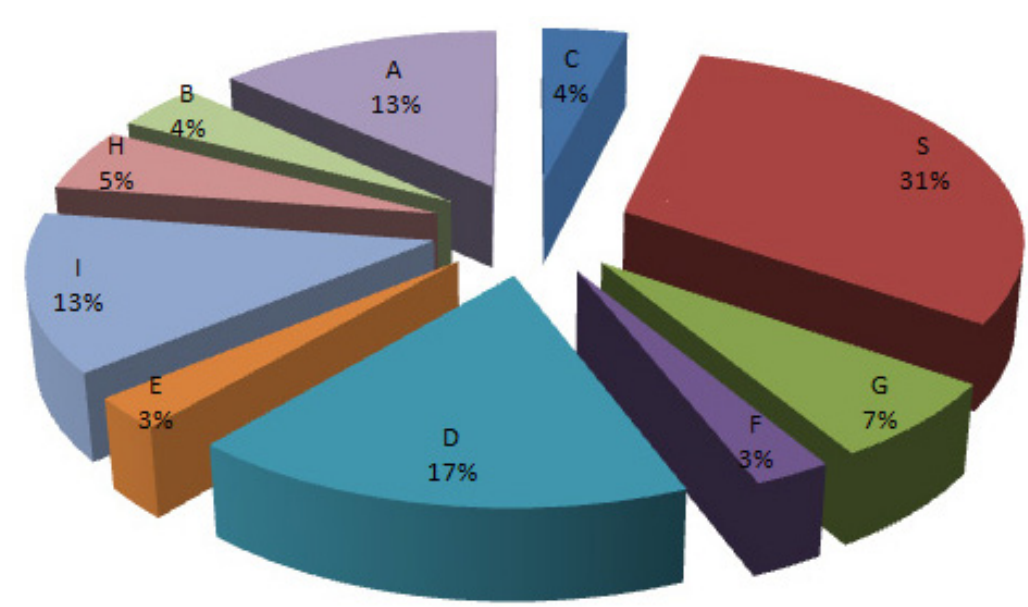

Figure 2. Classification of bZIP family TFs in carrot and the percentage of every subfamily.

\section{Motif analysis of the carrot bZIP TF family}

We deciphered the conserved motifs in the 75 bZIP TFs in carrot using MEME (Version 4.9.1). The logos of these motifs are listed in Figure 3. The constituent motifs of each TF in the bZIP family are shown in Figure 4. Motif 1 appeared in all the members, suggesting that it is a basic motif with a significant function in bZIP TFs. Motif 2 was found only in the members of group $D$ and in a single member of group S (Dck22667). Motifs 4, 6, and 7 were exclusive to the group D while Motifs 5, 8, and 9 appeared only in the group A. The constituents of groups A and D were abundant compared to the remaining subfamilies, which implied that the groups $A$ and $D$ were special among the subfamilies of bZIP family TFs in carrot. The factors Dck48330 and Dck27084, unlike other members of group A, exhibited only Motif 1, indicating that these two TF might have fewer functions than the other members of the bZIP family in carrot.

\section{Evolution of bZIP TFs among different plant species}

We analyzed the bZIP TF family and group A members of the bZIP family among different plants. Among 42 selected plant species, the gene sequences of group A bZIP family members of only Ricinus communis (Jin et al., 2014), Vitis vinifera (Liu et al., 2014), Solanum lycopersicum (Xu et al., 2013), and Hordeum vulgare (Pourabed et al., 2015) have been published. In addition, there are few reports on genome-wide studies on the bZIP TF family in other selected plants. These studies used the same process adopted for carrot in the current study to examine the number of bZIP TFs and group A members (Figure 5). The number of bZIP TFs in algae and land plants differed remarkably. The number of TFs in algae was less than 20 , while that in the land plants was greater than 25. The number of group A members in algae, bryophyte, pteridophyta, and gymnosperms was less than 5. No group A member was found in Ostreococcus lucimarinus. On the contrary, the number of group A members in land plants, except Picea abies, Selaginella moellendorffii, and Physcomitrella patens was greater than 5 . The average number of bZIP family TFs per Mb in $P$. abies, Arabidopsis thaliana, and carrot was $0.002,1.016$, and 0.156 , respectively. Among the selected plant species, the average number of bZIP family TFs per Mb was the highest in $A$. thaliana. 


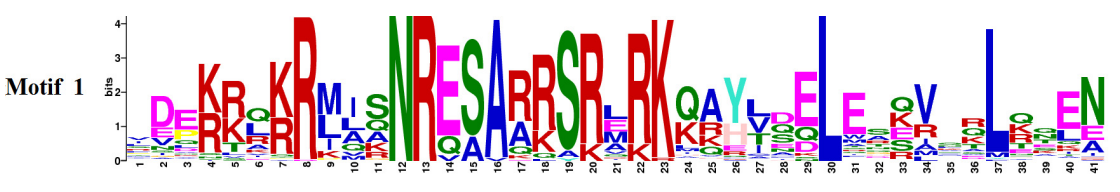

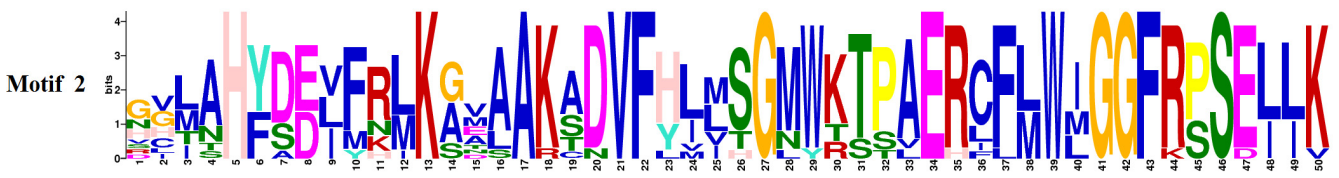

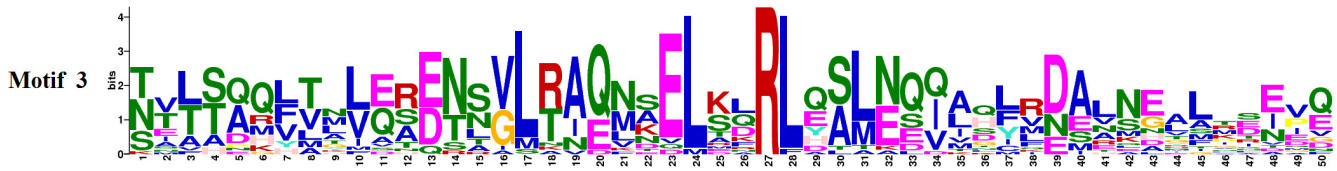

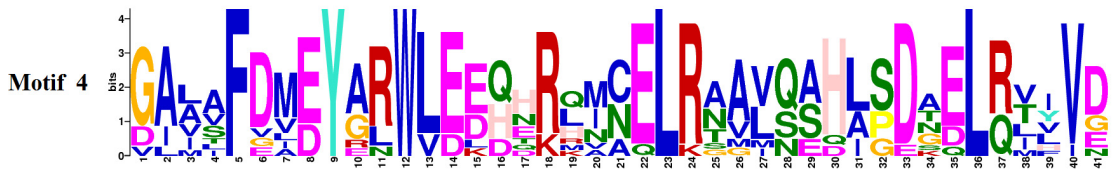

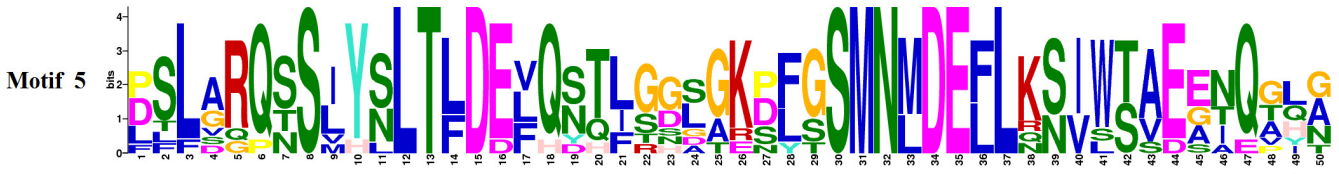

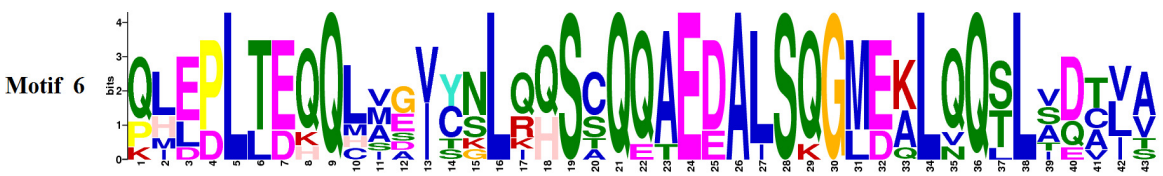

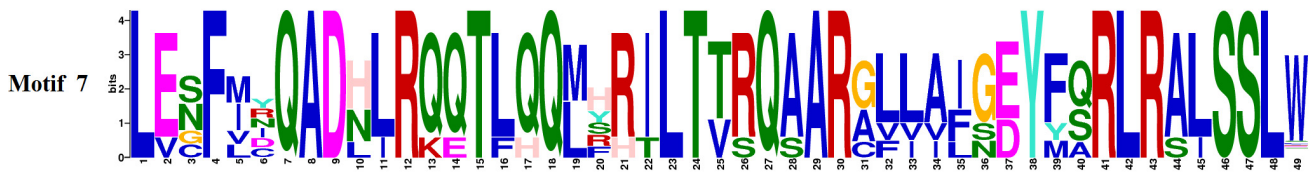
Motif 8 告-MGSR

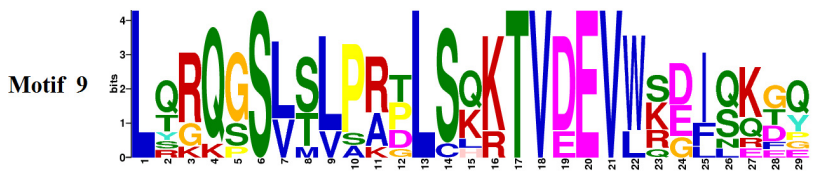

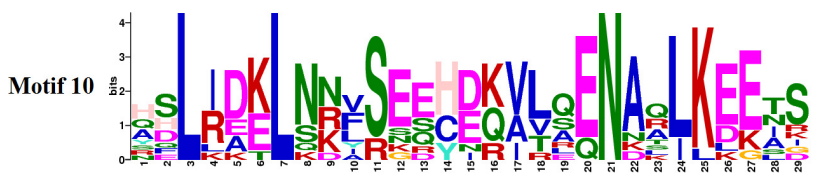

Figure 3. Sequence logos of bZIP TF domains in carrot. 


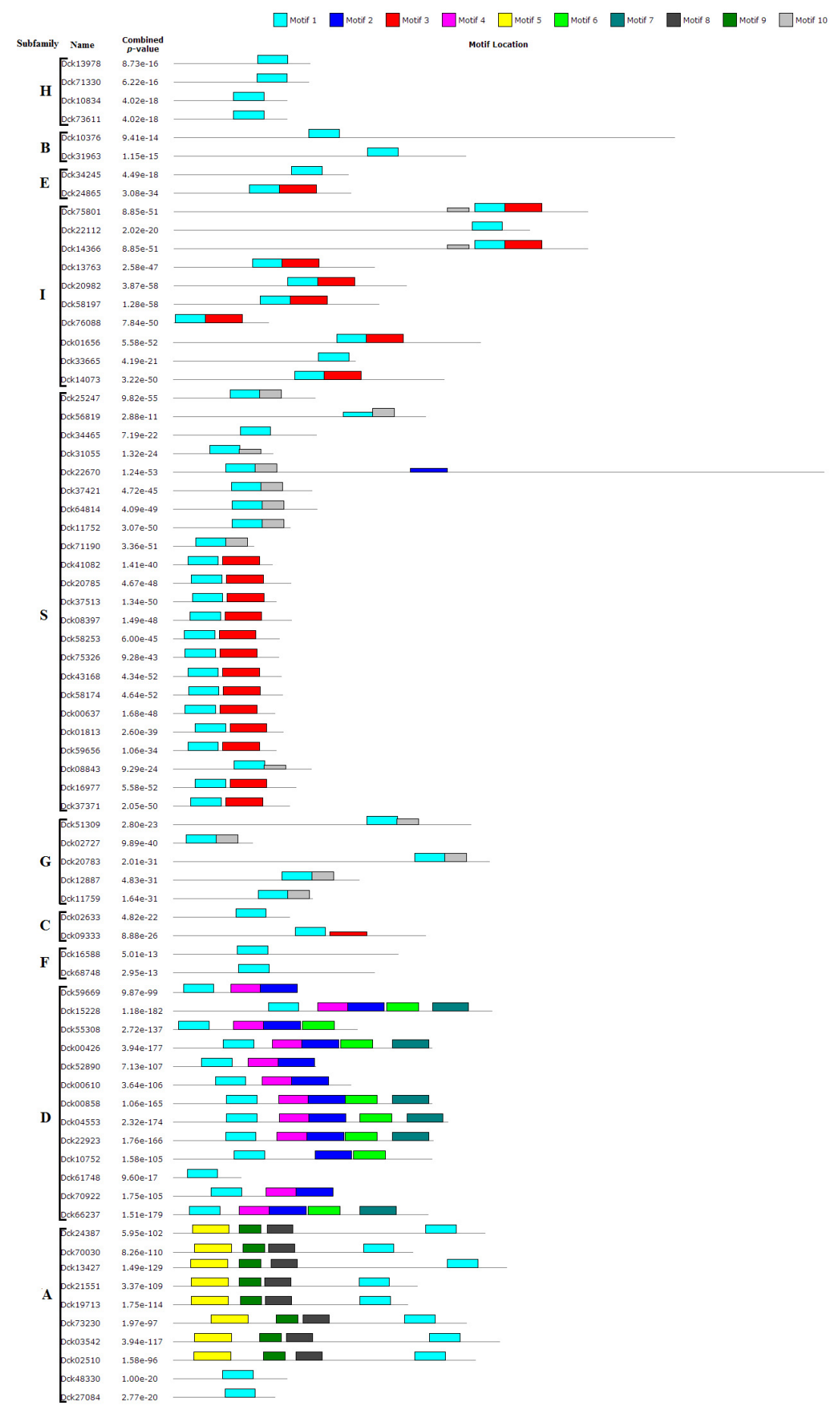

Figure 4. Motifs of bZIP TFs from different subfamilies in carrot. 


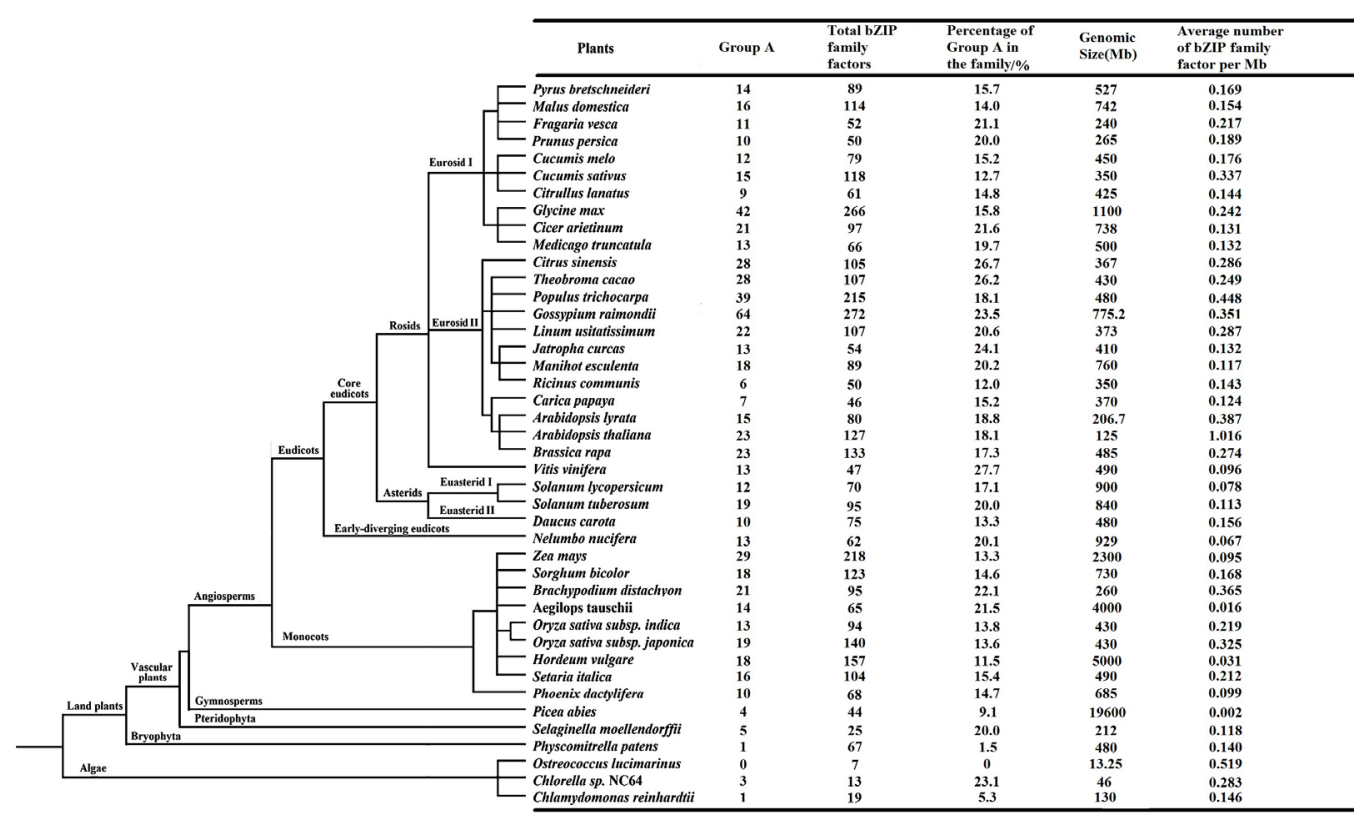

Figure 5. Evolution of bZIP family TFs among plants.

\section{Analysis of cis-regulatory elements}

We examined the upstream sequences of genes from the group $A$, in carrot, based on their genome sequences. The region 900-1500 bp upstream of the gene was investigated in all the cases except for DcbZIPA3, for which only the region 473 bp upstream was considered. A number of cis-regulatory elements were identified when a search was made for $b Z I P$ genes in the PlantCARE database. We selected 11 elements related to abiotic stress and hormone responsiveness for further investigations (Figure 6). Almost all members of group A exhibited the cis-regulatory elements involved in abiotic stress (i.e., drought, heat, low temperature) or defense and stress responsiveness. The cis-regulatory elements involved in hormone (i.e., abscisic acid, gibberellins, ethylene, MeJA, salicylicacid, and auxin) responsiveness were identified in DcbZIPA1 and DcbZIPA4-10. Among the 10 genes studied, seven had drought responsive elements, two had heat responsive elements, and only DcbZIPA10 showed the low-temperature responsive element. The numbers of cis-acting elements in DcbZIPA2, 3, and 9 were low, contrary to the large numbers observed in DcbZIPA5 and 6. Furthermore, three genes (DcbZIPA1, DcbZIPA5 and 7) had one ABRE element, while DcbZIPA6 had four.

\section{Prediction of bZIP protein (group A) folding state}

The folding state of the group A bZIP proteins was predicted via the Foldlndex program. The prediction results revealed disordered regions in the selected proteins gathered at the end of the ORF domain (Figure 7). Table 2 shows the specific data for the proteins. The percentages of disordered residues in DcbZIPA1, 2, and 5 were less than $50 \%$, while those of the remaining members of group A bZIP proteins were more than $50 \%$. The charge values of these proteins 
ranged from 0.000 to 0.071 . The phobic values of the proteins were uniform and ranged from 0.373 to 0.429 , with little difference between them.
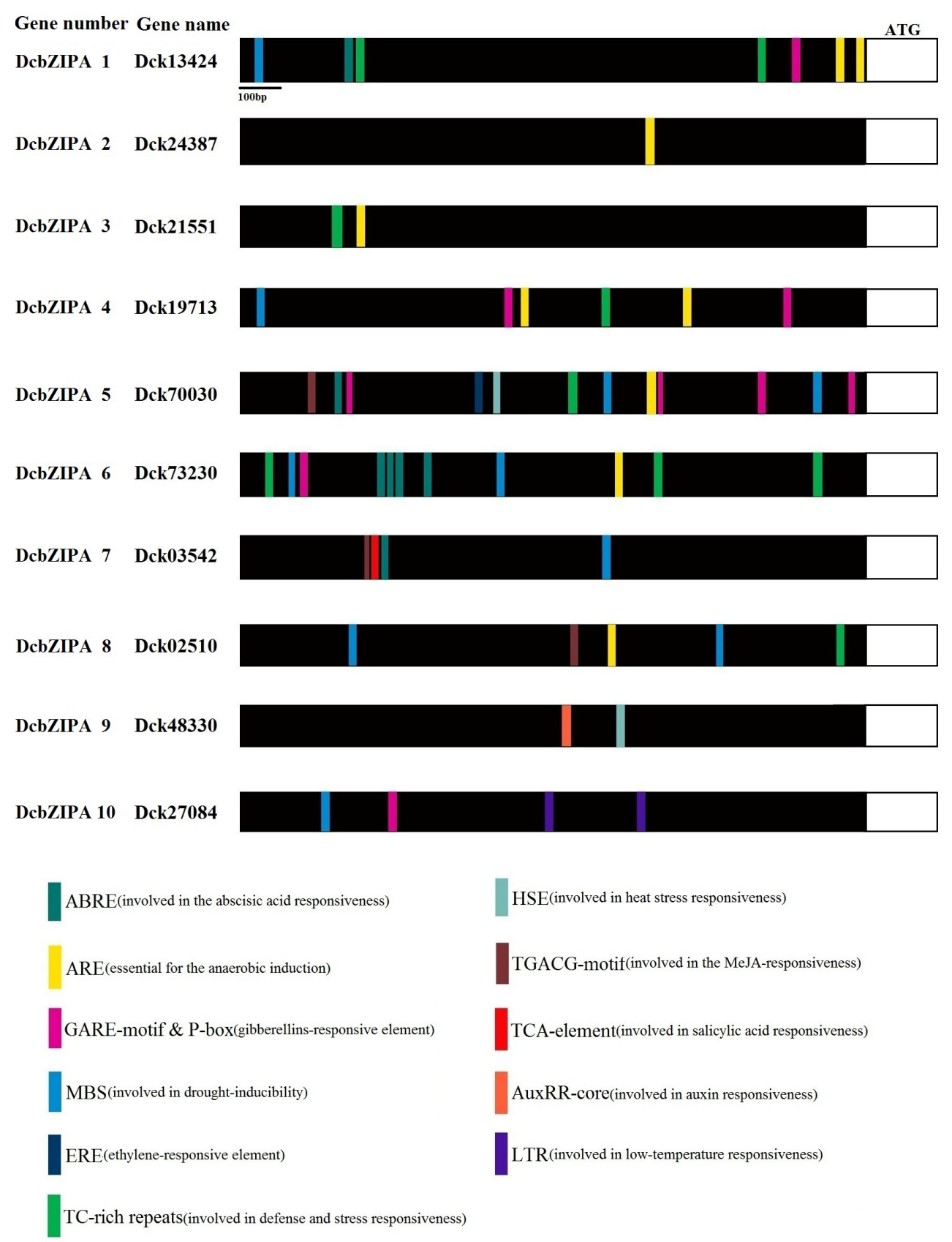

Figure 6. Regulatory regions of carrot bZIP genes. The 5' upstream regions of the bZIP genes are displayed. Elements involved in the different physiological processes are marked in different colors. 

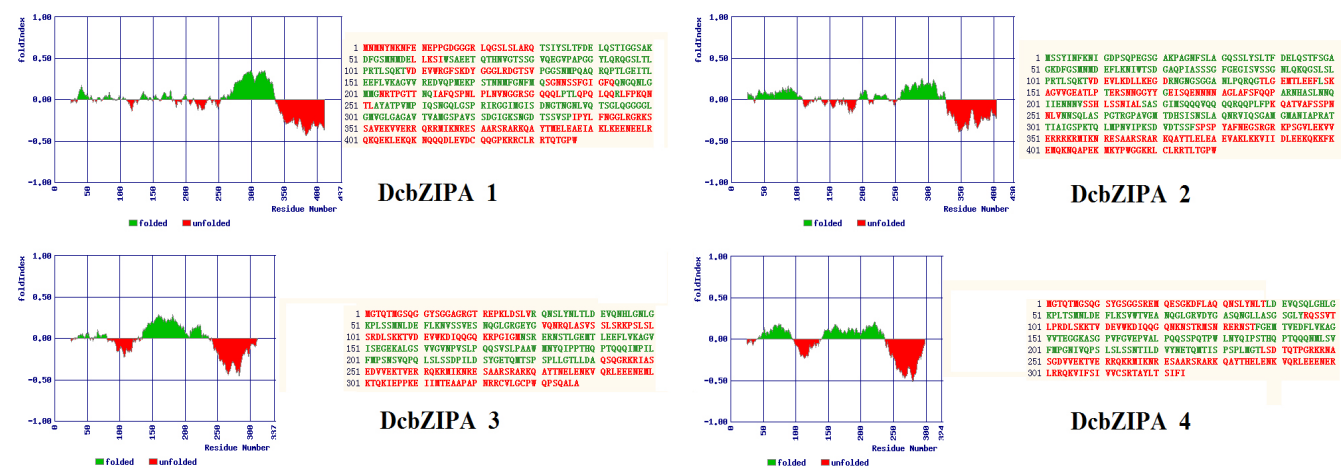

DcbZIPA 3
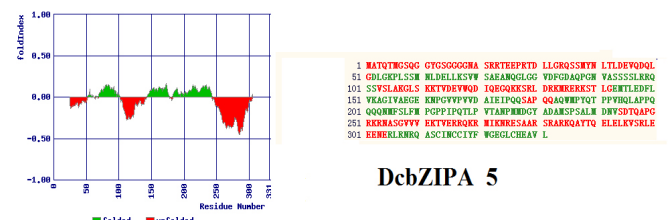

DcbZIPA 5
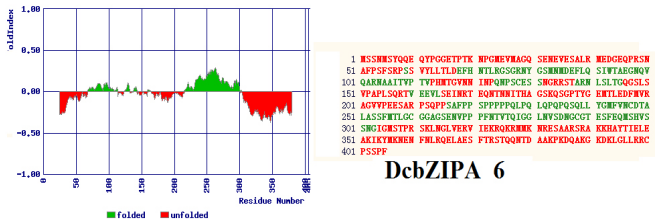

DcbZIPA 4
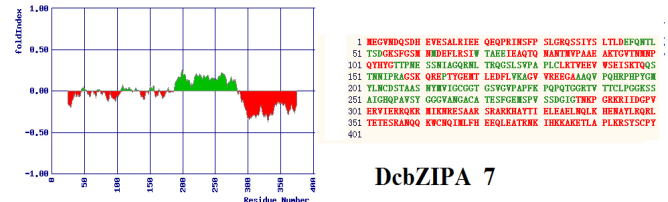

DcbZIPA 7

mfolded $=$ unfolder
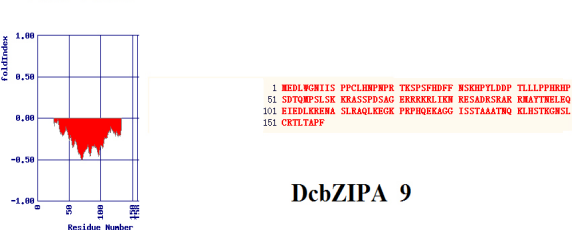

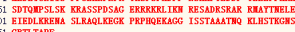

DcbZIPA 9
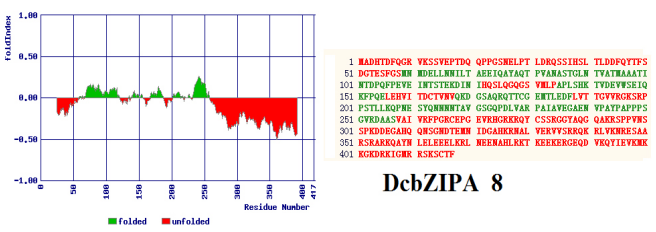

DcbZIPA 8

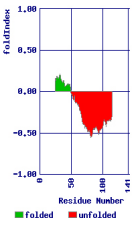

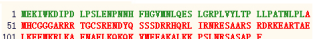

DcbZIPA 10

Figure 7. Prediction of the folding state of group A members of bZIP TFs. Ordered regions of the protein are marked in green, and disordered regions are in red. Relative amino acid residue sequences are also marked in green (ordered) and red (disordered).

Table 2. Prediction of the folding states of amino acid sequences of group A members of bZIP factors from $D$. carota.

\begin{tabular}{|c|c|c|c|c|c|c|c|}
\hline Factor number & $\begin{array}{c}\text { No. of } \\
\text { amino acid }\end{array}$ & $\begin{array}{l}\text { No. of disordered } \\
\text { regions }\end{array}$ & $\begin{array}{l}\text { Longest disordered } \\
\text { region }\end{array}$ & $\begin{array}{l}\text { No. of disordered } \\
\text { residues }\end{array}$ & $\begin{array}{c}\text { Percentage of } \\
\text { disordered residues (\%) }\end{array}$ & $\begin{array}{l}\text { Charge } \\
\text { value }\end{array}$ & $\begin{array}{l}\text { Phobic } \\
\text { value }\end{array}$ \\
\hline DcbZIPA 1 & 460 & 9 & 101 & 213 & 46.3 & 0.021 & 0.420 \\
\hline DcbZIPA 2 & 430 & 8 & 104 & 191 & 44.4 & 0.030 & 0.429 \\
\hline DcbZIPA 3 & 337 & 3 & 97 & 173 & 51.3 & 0.015 & 0.427 \\
\hline DcbZIPA 4 & 324 & 3 & 87 & 167 & 51.5 & 0.015 & 0.428 \\
\hline DcbZIPA 5 & 331 & 4 & 61 & 157 & 47.4 & 0.000 & 0.426 \\
\hline DcbZIPA 6 & 405 & 7 & 102 & 250 & 61.7 & 0.000 & 0.417 \\
\hline DcbZIPA 7 & 451 & 9 & 114 & 244 & 54.1 & 0.003 & 0.418 \\
\hline DcbZIPA 8 & 417 & 5 & 160 & 256 & 61.3 & 0.007 & 0.404 \\
\hline DcbZIPA 9 & 158 & 1 & 158 & 158 & 100.0 & 0.063 & 0.373 \\
\hline DcbZIPA10 & 141 & 1 & 92 & 92 & 65.2 & 0.071 & 0.386 \\
\hline
\end{tabular}




\section{qRT-PCR analysis of the expression of selected bZIP genes under abiotic stress}

The expression genes encoding the group A members of bZIP family in response to various abiotic stresses (i.e., low temperature, salt, drought, and heat) were assessed by qRT-PCR. Among the 10 selected genes, the expression of DcbZIPA 1, 3, 4, 5, 9, and 10 was clearly detected, that of DcbZIPA2, 6, and 8 was only partly detected, whereas for DcbZIPA7 it was undetectable (Figure 8).
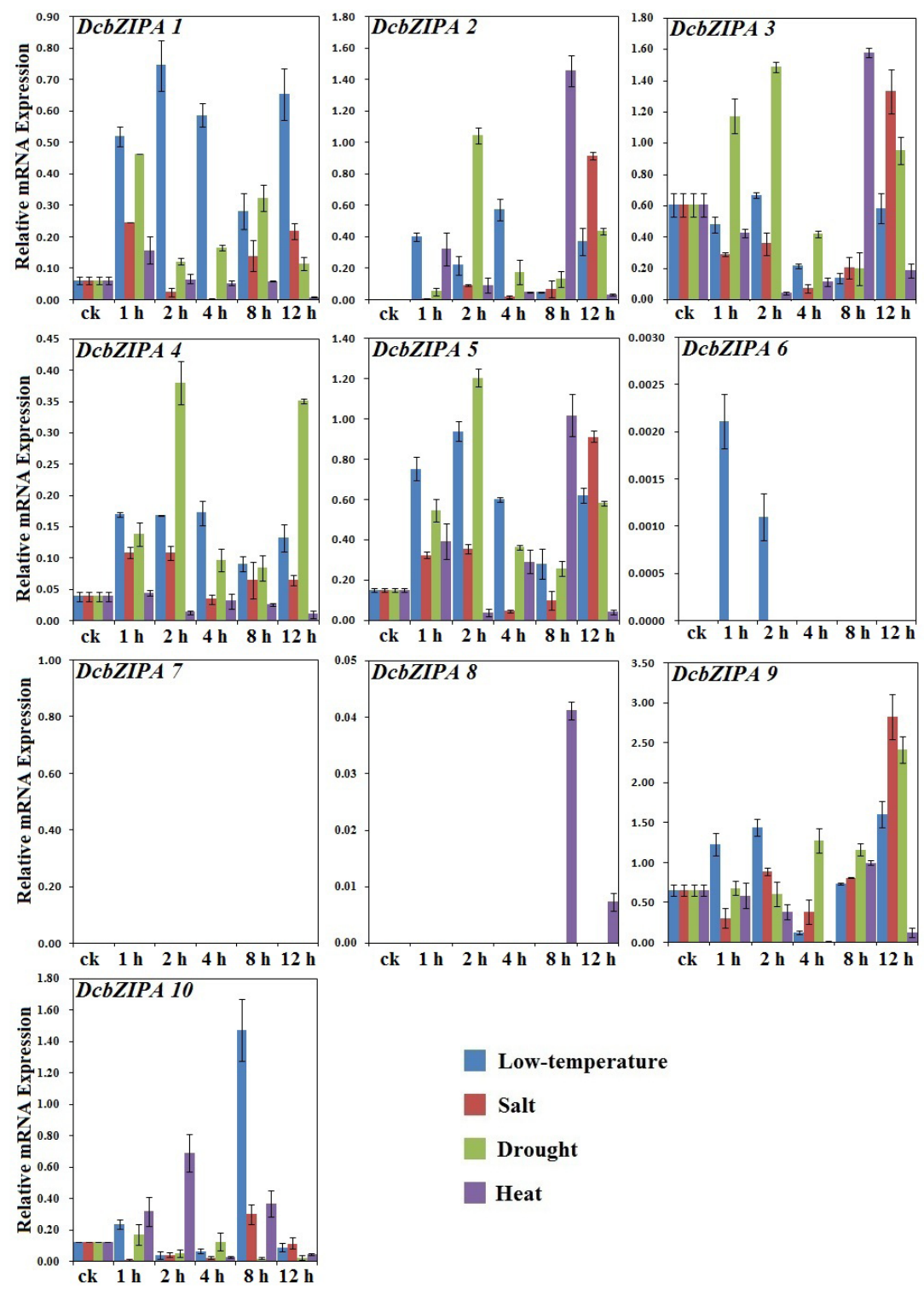

Low-temperature

Salt

Drought

Heat

Figure 8. Expression profiling of the $10 \mathrm{bZIP}$ genes of group A under abiotic stress. The mRNA level of Tubulin was defined as 1. Experiments were repeated thrice using independent RNA samples. Error bars were calculated based on three replicates. 


\section{Low-temperature treatment}

Eight genes (DcbZIPA1-6, 9, and 10) responded to the cold stress. DcbZIPA1, 2, 4, and 5 were upregulated after cold treatment. The expression of DcbZIPA2 was not detected in control check (CK). It was only detected after the cold treatment. The expression of DcbZIPA6 was detected only 1 and $2 \mathrm{~h}$ after the treatment while that of DcbZIPA7 and 8 were not detected.

\section{Salt treatment}

Seven genes (DcbZIPA1-5, 9, and 10) responded to the salt stress while the expressions of 3 genes (DcbZIPA6-8) were not detected. The expression of DcbZIPA2 was not observed in CK but it peaked $12 \mathrm{~h}$ after salt treatment.

\section{Drought treatment}

In response to drought treatment, seven genes (DcbZIPA1-5, 9, and 10) exhibited varied expressions. DcbZIPA1, 4, and 5 were upregulated after drought treatment. The expression of DcbZIPA2 was not detected in CK but was detected after PEG treatment $(200 \mathrm{~g} / \mathrm{L})$ and peaked at $2 \mathrm{~h}$ after the treatment. The expression of DcbZIPA6-8 was not observed under the drought stress.

\section{Heat treatment}

In response to heat treatment, eight genes (DcbZIPA1-5, 7, 9, and 10) exhibited varying expression. The expression of DcbZIPA8 was detected only after 8 and $24 \mathrm{~h}$ of the heat treatment. The expression of DcbZIPA2 was not detected in CK but was detected after treatment and peaked at $8 \mathrm{~h}$.

\section{DISCUSSION}

\section{Group A members of bZIP TFs in carrot}

bZIP TFs are important in the response of plants to abiotic stress (Yáñez et al., 2009). In Arabidopsis, this TF family has been divided into ten (A-I and S) groups according to their DNAbinding domain (Jakoby et al., 2002). The bZIP family of $D$. carota has also been classified into 10 subfamilies; 10 out of 75 members of this family belong to the group A. Analysis of the motifs of this family supports this classification.

\section{Evolution of bZIP TFs among plant species}

During evolution, the plant genome must change to adapt to environmental variations and become more complex. Plant development and growth depends on the proper regulation of several genes (Cheon et al., 2011). bZIP TFs have been reported to play vital roles in these regulation processes (Wray et al., 2003). Comparison of the genome size of algae and land plants, in this study, showed that their genome sizes are drastically different. The number of bZIP factors was also considerably different between algae and land plants. The number of group A bZIP factors in land plants was obviously larger than that in algae, except for $P$. abies, S. moellendorffii, and $P$. patens. These results indicate that the group A bZIP TFs are associated with plant evolution and 
perform key roles in the process. Compared to those of other plants, the genome size of carrot (Apiaceae, $480 \mathrm{Mb}$; Xu et al., 2014) is about 4 times that of the model plant, A. thaliana (125 $\mathrm{Mb}$ ) (Arabidopsis Genome Initiative, 2000) and similar to those of rice (Oryza sativa ssp. indica, $430 \mathrm{Mb}$;Yu et al., 2002) and grapevine (Vitis vinifera,487 Mb;Jaillon et al., 2007). The average number of bZIP family TFs per Mb in A. thaliana, rice (ssp. indica), grapevine, and carrot was $1.016,0.219,0.096$, and 0.156 , respectively. In plants, bZIP TFs appeared before the origin of algae and have been duplicated many times during plant evolution. bZIP TFs might thus have expanded during plant evolution.

\section{Group A members of bZIP TFs among plants}

In plants, $A B A$ is an important factor mediating their response to abiotic stresses (Gorji et al., 2013). The phytohormone ABA takes part in the regulation of many stress-inducing genes, including some bZIP TFs (Mehrotra et al., 2014). A number of group A bZIP TFs have been reported to function in ABA signaling (Fujita et al., 2011). In Arabidopsis, AtbZIP39, 36, and 38 are reported to function in ABA signaling (Choi et al., 2000; Finkelstein and Lynch, 2000; Uno et al., 2000; Lopez-Molina et al., 2001). In O. sativa, similar reports on OsABF1 (Hossain et al., 2010b), OsABI5 (Zou et al., 2008), and OsABF2 are available (Hossain et al., 2010a). ABRE is reported to be the main cis-acting regulatory element in ABA-responsive gene expression (Hossain et al., 2010a). Analysis of the cis-regulatory elements of the 10 genes encoding DcbZIPA in carrot revealed that four (DcbZIPA1, DcbZIPA5-7) had ABRE elements. These genes might perform important functions in ABA signaling in carrot.

\section{Response of group A members to abiotic stress}

Abiotic stress is a serious limiting factor during carrot production. Drought, high salt, heat, and low temperature are the main abiotic stresses severely affecting carrot production (Shinozaki et al., 2003; Chauhan et al., 2013). In this study, 2-month-old carrot plants were subjected to high $\left(38^{\circ} \mathrm{C}\right)$ and low temperature $\left(4^{\circ} \mathrm{C}\right)$, high salt $(0.2 \mathrm{M} \mathrm{NaCl})$, and dehydration $(200 \mathrm{~g} / \mathrm{L} \mathrm{PEG})$ stress. The expression of the 10 selected genes was analyzed by qRT-PCR. Expression of seven of these genes was detected after the treatment. The expression of other genes (DcbZIPA6-8) was either not detected or only partly detected. The expression of DcbZIPA2 was unique because it could not be detected in CK but was detected after the treatment.

The study of protein intrinsic disorder (ID) is an emerging field in basic plant science (Dunker et al., 2002). ID is reported to appear in many highly regulated pathways that can influence the DNA binding (Kragelund et al., 2012). According to a previous study, ID is a feature of whole proteins resulting from a high number of charged amino acid residues and low hydrophobicity (Dunker et al., 2001). To examine the group A members of the bZIP family in carrot further, we predicted the folding state of all members of the subfamily. We found three genes, i.e., DcbZIPA6-8, the expressions of which were either not detected or only partially detected and differed from the others in terms of several indices. The percentages of disordered residues in these genes were higher than those of the other genes, except for DcbZIPA9 and 10; their charge values were also lower. During analysis of cis-elements, these three genes revealed several elements upstream of their ORFs that are associated with abiotic stresses. However, the expression of the three genes was not detected after the treatments. The folding state of the proteins expressed by these genes might probably be associated with this phenomenon. 


\section{CONCLUSIONS}

The group A of bZIP family TFs is important in the evolution of plants. Carrot genome harbors 75 genes encoding the bZIP family TFs; 10 of these are classified into group A. The modulation of expression of these genes under abiotic stresses (salt, drought, cold, and heat) suggests that this group is widely involved in the response to abiotic stress in carrot. The behavior of four 'special' members as discussed above needs further study to find a plausible explanation. The $D$. carota genome sequence offers a significant resource for analyzing the bZIP TFs. The results presented in this study will facilitate further study on the group A bZIP TFs regarding their functions in response to abiotic stresses.

\section{ACKNOWLEDGMENTS}

Research supported by the New Century Excellent Talents in University (\#NCET-110670), Jiangsu Natural Science Foundation (\#BK20130027), and Priority Academic Program Development of Jiangsu Higher Education Institutions Project (PAPD).

\section{REFERENCES}

Arabidopsis Genome Initiative (2000). Analysis of the genome sequence of the flowering plant Arabidopsis thaliana. Nature 408: 796-815.

Bailey TL, Williams N, Misleh C and Li WW (2006). MEME: discovering and analyzing DNA and protein sequence motifs. Nucleic. Acids Res. 34: W369-W373.

Chauhan H, Khurana N, Agarwal P, Khurana JP, et al. (2013). A seed preferential heat shock transcription factor from wheat provides abiotic stress tolerance and yield enhancement in transgenic Arabidopsis under heat stress environment. PloS One 8: e79577.

Chen W, Provart NJ, Glazebrook J, Katagiri F, et al. (2002). Expression profile matrix of Arabidopsis transcription factor genes suggests their putative functions in response to environmental stresses. Plant Cell 14: 559-574.

Cheon SA, Jung KW, Chen YL, Heitman J, et al. (2011). Unique evolution of the UPR pathway with a novel bZIP transcription factor, Hxl1, for controlling pathogenicity of Cryptococcus neoformans. PLoS Pathogens 7: e1002177. doi:10.1371/ journal.ppat.1002177.

Choi H, Hong J, Ha J, Kang J, et al. (2000). ABFs, a family of ABA-responsive element binding factors. J. Biol. Chem. 275: 1723-1730.

Dunker AK, Brown CJ, Lawson JD, lakoucheva LM, et al. (2002). Intrinsic disorder and protein function. Biochemistry 41: 6573-6582.

Dunker AK, Lawson JD, Brown CJ, Williams RM, et al. (2001). Intrinsically disordered protein. J. Mol. Graph. Model. 19: $26-59$.

Ellenberger TE, Brandl CJ, Struhl K and Harrison SC (1992). The GCN4 basic region leucine zipper binds DNA as a dimer of uninterrupted $\alpha$ helices: crystal structure of the protein-DNA complex. Cell 71: 1223-1237.

Finkelstein RR and Lynch TJ (2000). The Arabidopsis abscisic acid response gene ABI5 encodes a basic leucine zipper transcription factor. Plant Cell 12: 599-609.

Fujita Y, Fujita M, Satoh R, Maruyama K, et al. (2005). AREB1 is a transcription activator of novel ABRE-dependent ABA signaling that enhances drought stress tolerance in Arabidopsis. Plant Cell 17: 3470-3488.

Fujita Y, Fujita M, Shinozaki K and Yamaguchi-Shinozaki K (2011). ABA-mediated transcriptional regulation in response to osmotic stress in plants. J. Plant Res. 124: 509-525.

Gorji A, Hajianfar R and Rostamforody B (2013). Effects of cold and other abiotic stress on plants. Intl. J. Agron. Plant Prod. 4: 3597-3604.

Hossain MA, Cho JI, Han M, Ahn CH, et al. (2010a). The ABRE-binding bZIP transcription factor OsABF2 is a positive regulator of abiotic stress and ABA signaling in rice. J. Plant Physiol. 167: 1512-1520.

Hossain MA, Lee Y, Cho J-I, Ahn C-H, et al. (2010b). The bZIP transcription factor OsABF1 is an ABA responsive element binding factor that enhances abiotic stress signaling in rice. Plant Mol. Biol. 72: 557-566.

Hsieh TH, Li CW, Su RC, Cheng CP, et al. (2010). A tomato bZIP transcription factor, SIAREB, is involved in water deficit and salt stress response. Planta 231: 1459-1473. 
Jaillon O, Aury JM, Noel B, Policriti A, et al. (2007). The grapevine genome sequence suggests ancestral hexaploidization in major angiosperm phyla. Nature 449: 463-467.

Jakoby M, Weisshaar B, Dröge-Laser W, Vicente-Carbajosa J, et al. (2002). bZIP transcription factors in Arabidopsis. Trends Plant Sci. 7: 106-111.

Jin Z, Xu W and Liu A (2014). Genomic surveys and expression analysis of bZIP gene family in castor bean (Ricinus communis L.). Planta 239: 299-312.

Kragelund BB, Jensen MK and Skriver K (2012). Order by disorder in plant signaling. Trends Plant Sci. 17: 625-632.

Lescot M, Déhais $P$, Thijs G, Marchal K, et al. (2002). PlantCARE, a database of plant cis-acting regulatory elements and a portal to tools for in silico analysis of promoter sequences. Nucleic Acids Res. 30: 325-327.

Liu J, Chen N, Chen F, Cai B, et al. (2014). Genome-wide analysis and expression profile of the bZIP transcription factor gene family in grapevine (Vitis vinifera). BMC Genomics 15: 281. doi:10.1186/1471-2164-15-281.

Lopez-Molina L, Mongrand S and Chua N-H (2001). A postgermination developmental arrest checkpoint is mediated by abscisic acid and requires the ABI5 transcription factor in Arabidopsis. Proc. Natl. Acad. Sci. USA 98:4782-4787.

Mehrotra R, Bhalothia P, Bansal P, Basantani MK, et al. (2014). Abscisic acid and abiotic stress tolerance-different tiers of regulation. J. Plant Physiol. 171: 486-496.

Pourabed E, Ghane Golmohamadi F, Soleymani Monfared P, Razavi SM, et al. (2015). Basic leucine zipper family in barley: genome-wide characterization of members and expression analysis. Mol. Biotechnol. 57: 12-26.

Prilusky J, Felder CE, Zeev-Ben-Mordehai T, Rydberg EH, et al. (2005). Foldlndex: a simple tool to predict whether a given protein sequence is intrinsically unfolded. Bioinformatics 21: 3435-3438.

Riechmann JL, Heard J, Martin G, Reuber L, et al. (2000). Arabidopsis transcription factors: genome-wide comparative analysis among eukaryotes. Science 290: 2105-2110.

Shinozaki K, Yamaguchi-Shinozaki K and Seki M (2003). Regulatory network of gene expression in the drought and cold stress responses. Curr. Opin. Plant Biol. 6: 410-417.

Tamura K, Peterson D, Peterson N, Stecher G, et al. (2011). MEGA5: molecular evolutionary genetics analysis using maximum likelihood, evolutionary distance, and maximum parsimony methods. Mol. Biol. Evol. 28: 2731-2739.

Thompson JD, Gibson TJ, Plewniak F, Jeanmougin F, et al. (1997). The CLUSTAL_X windows interface: flexible strategies for multiple sequence alignment aided by quality analysis tools. Nucleic Acids Res. 25: 4876-4882.

Uno Y, Furihata T, Abe H, Yoshida R, et al. (2000). Arabidopsis basic leucine zipper transcription factors involved in an abscisic acid-dependent signal transduction pathway under drought and high-salinity conditions. Proc. Natl. Acad. Sci. USA97: 11632-11637.

Vinocur B and Altman A (2005). Recent advances in engineering plant tolerance to abiotic stress: achievements and limitations Curr. Opin. Biotechnol. 16: 123-132.

Wang J, Zhou J, Zhang B, Vanitha J, et al. (2011). Genome-wide expansion and expression divergence of the basic leucine zipper transcription factors in higher plants with an emphasis on sorghum. J. Integr Plant Biol. 53: 212-231.

Wray GA, Hahn MW, Abouheif E, Balhoff JP, et al. (2003). The evolution of transcriptional regulation in eukaryotes. Mol. Biol. Evol. 20: 1377-1419.

Xu R, Zhang S, Lu L, Cao H, et al. (2013). A genome-wide analysis of the RNA helicase gene family in Solanum lycopersicum. Gene 513: 128-140.

Xu ZS, Tan HW, Wang F, Hou XL, et al. (2014). CarrotDB: a genomic and transcriptomic database for carrot. Database (Oxford) 2014: bau096.

Yáñez M, Cáceres S, Orellana S, Bastías A, et al. (2009). An abiotic stress-responsive bZIP transcription factor from wild and cultivated tomatoes regulates stress-related genes. Plant Cell Rep. 28: 1497-1507.

Yu J, Hu S, Wang J, Wong GK, et al. (2002). A draft sequence of the rice genome (Oryza sativa L. ssp. indica). Science $296:$ 79-92.

Zou M, Guan Y, Ren H, Zhang F, et al. (2008). A bZIP transcription factor, OsABI5, is involved in rice fertility and stress tolerance. Plant Mol. Biol. 66: 675-683. 